\title{
NOUVELLE
}

\section{Caresser la polarité planaire dans le sens du flot}

Benoît Aigouy, Raphaël Etournay, Andreas Sagner, Douglas B. Staple, Reza Farhadifar, Jens-Christian Röper, Suzanne Eaton, Frank Jülicher

B. Aigouy, R. Etournay, A. Sagner, J.C. Röper, S. Eaton : Max Planck institute of molecular cell biology and genetics, Pfotenhauerstrasse 108, Dresde 01307, Allemagne.

\section{aigouy@mpi-cbg.de}

D.B. Staple, R. Farhadifar, F. Jülicher :

Max Planck institute for the physics of complex systems, Noethnitzer strasse 38, Dresde 01187, Allemagne.
> La plupart des cellules épithéliales sont polarisées dans le plan de l'épithélium $[1,2]$. Cette polarité, appelée polarité planaire, est particulièrement visible
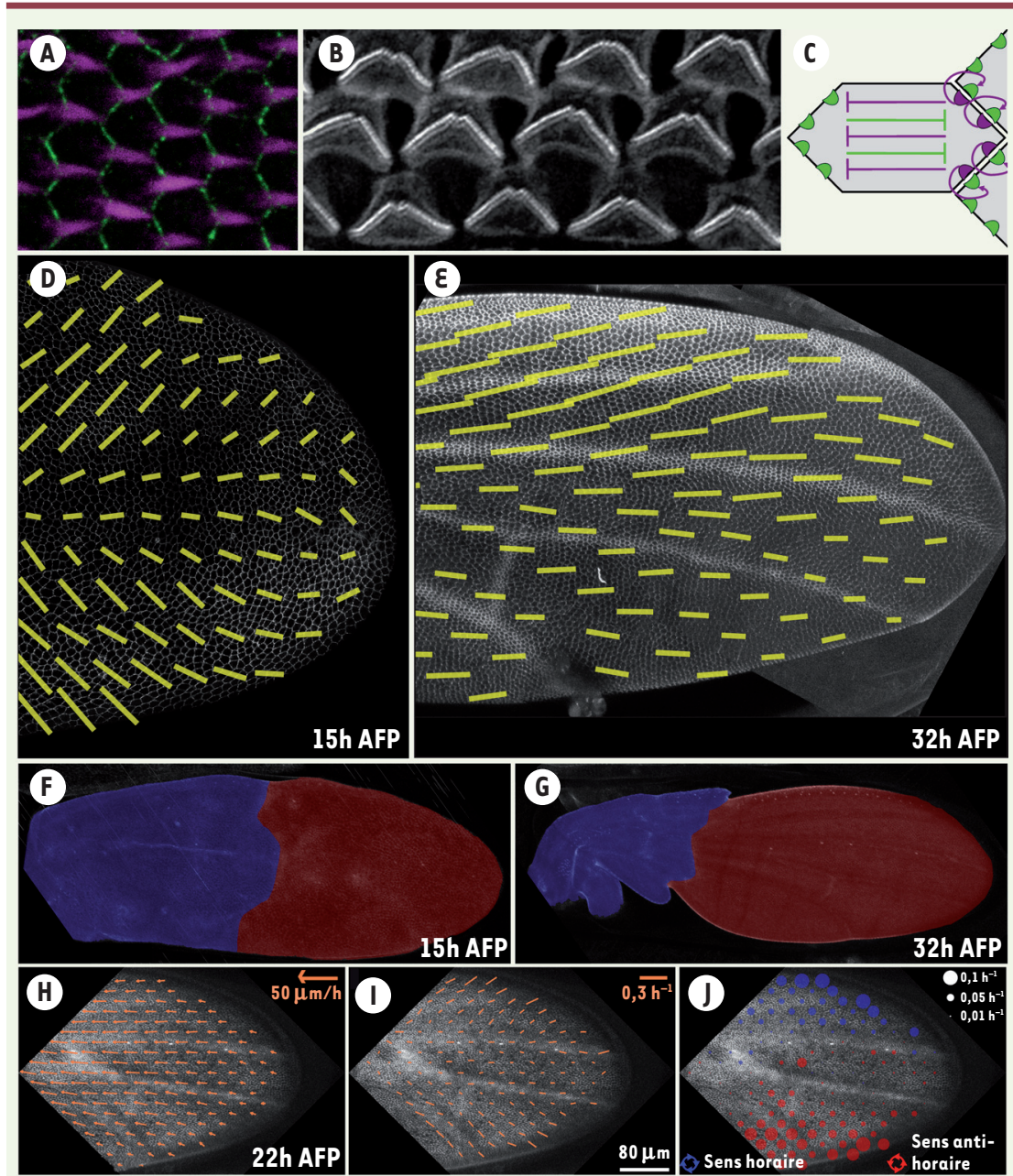

K
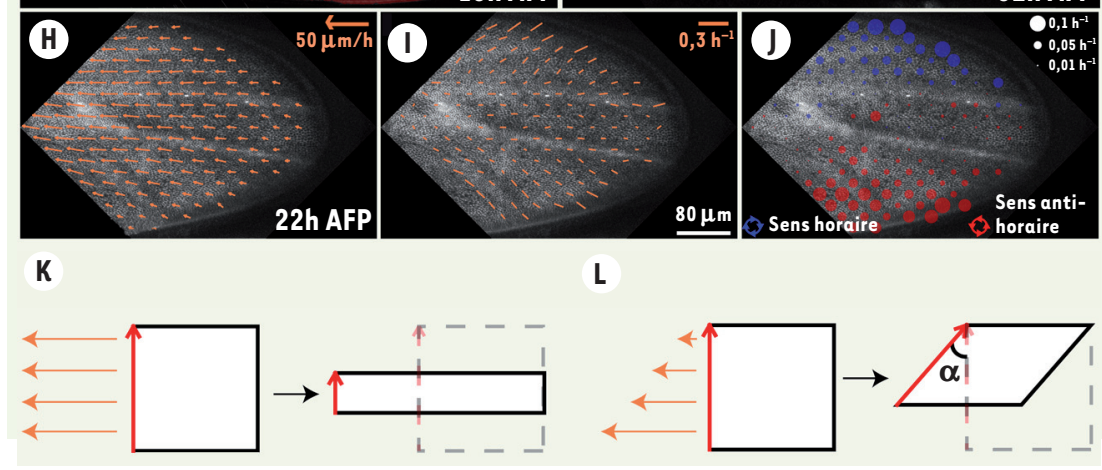

phile (Figure 1A, B). Dans ces deux cas, l'alignement des structures externes est contrôlé par les protéines de polarité planaire. Ces protéines ont été décrites

Figure 1. La polarité planaire se réoriente au cours du développement. A-B. Exemples de tissus polarisés dans le plan. $\boldsymbol{A}$. Immunomarquage d'une aile de drosophile après émergence des poils. Chaque cellule de l'aile produit un seul poil (violet) qui pointe du côté distal de l'aile. B. Cochlée de souris. Chaque cellule ciliée externe arbore une touffe ciliaire en forme de chevron. Ces chevrons pointent tous dans la même direction. C. Schéma de l'autorégulation des protéines de polarité planaire. Les domaines distaux (violets) et proximaux (verts) s'excluent intracellulairement et se recrutent entre cellules adjacentes. $\boldsymbol{D}-\boldsymbol{\varepsilon}$. Polarité planaire (segments jaunes) dans une aile de drosophile à 15 heures AFP (D) et à 32 heures $\operatorname{AFP}(\varepsilon)$. Chaque segment est obtenu en calculant la moyenne de la polarité d'un petit groupe de cellules. F-G. Changements morphologiques d'une aile de drosophile entre 15 heures AFP et 32 heures AFP. La partie proximale de l'aile est colorée en bleu et la partie distale en rouge. $\boldsymbol{H}$. La contraction de la partie proximale de l'aile induit un flot cellulaire non homogène dans la partie distale de l'aile. I-J. Ce flot génère des contraintes mécaniques locales de type cisaillement ( $I$ ) et rotation ( $J$ ). $\boldsymbol{K}$. Exemple de cisaillement pur causé par un flot homogène. L. Exemple de cisaillement simple causé par un flot non homogène : le cisaillement simple se décompose en un cisaillement pur (voir $\boldsymbol{K}$ ) accompagné d'une rotation ( $\alpha$ ). AFP : après formation de la pupe. 


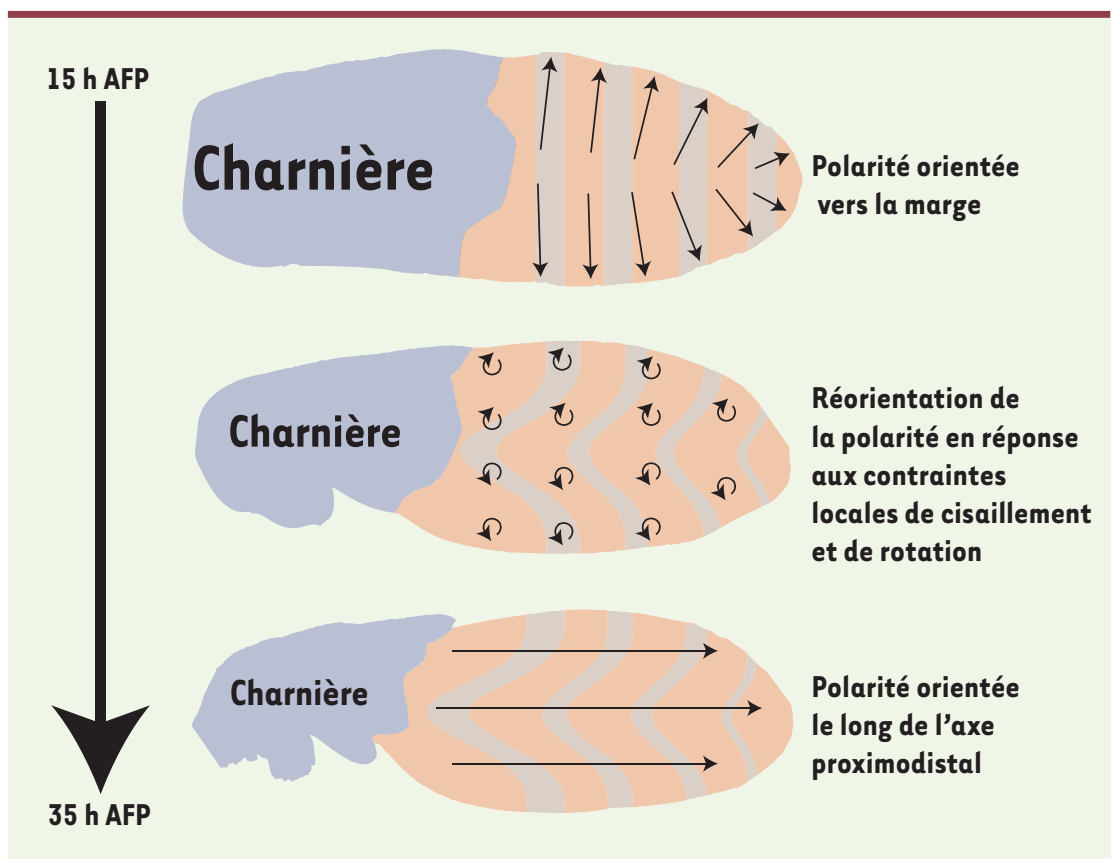

de manière approfondie dans l'aile de drosophile où elles s'auto-organisent en deux domaines distincts localisés asymétriquement dans chaque cellule, peu avant l'émergence des poils (Figure 1C). Un de ces domaines est localisé du côté proximal de la cellule et l'autre du côté distal. Cette ségrégation proximodistale (PD) est obtenue par exclusion mutuelle des domaines à l'intérieur d'une même cellule et par recrutement réciproque à l'interface entre deux cellules (Figure 1C) $[1,2]$. Ces interactions sont suffisantes pour expliquer l'alignement local de la polarité [4-6]. En revanche, elles n'expliquent pas comment les cellules peuvent aligner leur polarité globalement à l'échelle du tissu. C'est ce dernier point que nous aborderons ici.

L'axe de la polarité planaire pivote au cours du développement de l'aile Afin de comprendre comment la polarité évolue pendant le développement pupal, nous avons quantifié la distribution des protéines de polarité planaire entre 15 heures et 32 heures après formation de la pupe (AFP). À 15 heures AFP, l'axe de polarité planaire est orienté vers la marge de l'aile, formant un angle d'environ 45 degrés avec I'axe PD du tissu (Figure 1D) [7, 8]. Au cours du temps et jusqu'à 32 heures AFP, cet axe de polarité pivote et s'aligne sur l'axe PD [7] (Figure 1E). En conclusion, la polarité planaire ne se développe pas de novo, mais elle se réoriente au cours du développement.

\section{L'alignement}

de la polarité planaire est couplé aux changements morphologiques de l'aile Pendant la réorientation de la polarité planaire, l'épithélium subit d'importants changements morphologiques. La partie proximale de l'aile se contracte fortement (Figure IF, G), entraînant un flot cellulaire dans la partie distale (Figure $1 H$ ). Ce flot est plus rapide au centre qu'à la périphérie de l'aile (flot non homogène), ce qui crée des contraintes mécaniques locales de type cisaillement et rotation (Figure $1 /, J$ ). Lorsque la partie distale de l'aile est déconnectée de la partie proximale, le flot cellulaire, la forme de l'aile et l'alignement global de la polarité planaire sont perturbés [7]. Ces résultats indiquent que la réorientation de la polarité planaire est couplée aux contraintes mécaniques générées dans l'aile (rotation et cisaillement).
Figure 2. Modèle de la réorientation de la polarité planaire dans un flot non homogène. A. La polarité planaire (flèches noires) pointe initialement vers la marge de l'aile. $\boldsymbol{B}$. La partie proximale de l'aile (charnière) se contracte, entraînant des contraintes locales de type cisaillement et rotation dans la partie distale de l'aile. C. En réponse à ces contraintes, la polarité s'aligne le long de l'axe proximo-distal de l'aile. AFP : après formation de la pupe.

\section{Cisaillement et rotation entraînent} la réorientation de la polarité planaire La réorientation de polarité dans un flot non homogène a déjà été étudiée dans le cas des fluides complexes tels que les cristaux liquides polarisés et peut être décrite par l'équation phénoménologique suivante $[9,10]$ :

$$
\frac{d \theta}{d t}=\omega+v k_{s} \sin (2 \theta)
$$

Ici, la réorientation de l'axe de polarité $(d \theta / d t)$ dépend du taux de rotation locale $(\omega)$ et du taux de cisaillement pur $\left(k_{s}\right)$. Dans l'aile de drosophile, $\left(k_{s}\right)$ et $(\omega)$ peuvent être calculés à partir du flot cellulaire observé (voir Figure $1 H, J$ ). Le paramètre $v$, qui est inconnu, décrit la manière dont le cisaillement pur ${ }^{1}$ (Figure $1 K$ ) influence la réorientation de l'axe de polarité. Nous avons donc cherché à identifier les valeurs de $v$ permettant d'obtenir une rotation de polarité semblable à celle observée dans l'aile de drosophile. Nous avons d'abord considéré le cas $v=0$, pour lequel la réorientation de la polarité dépend uniquement des rotations locales et non du cisaillement pur. Dans ce cas, l'axe de polarité planaire pivote vers l'axe PD mais cette réorientation est incomplète [7]. Ce résultat indique que les rotations locales, à elles seules, ne suffisent pas à expliquer la réorientation observée expérimentalement et que le cisaillement pur doit lui aussi jouer un rôle (c'est-à-dire : $v \neq 0$ ).

${ }^{1}$ Le cisaillement pur résulte en une déformation du matériau sans aucune rotation de ses parois. 
Dans les cas où $v$ est positif, la polarité tend à s'aligner perpendiculairement à l'axe PD, à l'opposé de l'orientation observée dans l'aile. En revanche, pour un large éventail de valeurs négatives de $v$, la réorientation de la polarité prédite par l'équation (1) est en accord avec la réorientation observée dans l'aile (Figure 2). L'ensemble de ces résultats montre que la réorientation de la polarité est la conséquence des rotations locales et de l'alignement de la polarité dans la direction du cisaillement $(v<0)$.

\section{Conclusion}

Nous avons identifié un nouveau mécanisme coordonnant la polarité planaire avec la forme de l'aile. En particulier, nous avons décrit comment un flot cellulaire induit des contraintes mécaniques orientées qui concourent au pivotement de la polarité vers l'axe le plus long de l'aile (axe PD). Notre analyse théorique prédit aussi que le fait de changer la valeur de $v$, c'est-à-dire la manière dont le tissu répond au cisaille- ment, permettrait d'aligner la polarité perpendiculairement au cisaillement, dans l'axe court de l'épithélium, comme c'est le cas dans la cochlée. Enfin, nos travaux montrent l'existence et l'importance d'une polarité planaire initiale dont l'origine reste à déterminer. $\diamond$ Cell flow reorients planar polarity in the wing of Drosophila

\section{REMERCIEMENTS}

Les auteurs remercient Christine Petit pour la relecture de l'article et pour l'image de la cochlée dans la Figure 1. Ces travaux ont été soutenus par la Max Planck Gesellschaft, la Fondation pour la Recherche Médicale, le $7^{\text {th }}$ عU Framework Program, le Natural Sciences and Engineering Research Council of Canada, le Deutscher Akademischer Austausch Dienst et les Boehringer Ingelheim Fonds.

\section{CONFLIT D'INTÉRÊTS}

Les auteurs déclarent n'avoir aucun conflit d'intérêts concernant les données publiées dans cet article.

\section{RÉFÉRENCES}

1. Schweisguth $F$. Bases génétiques de la polarité planaire. Med Sci (Paris) 2004 ; 20 : 424-30.

2. Simons M, Mlodzik M. Planar cell polarity signaling: from fly development to human disease. Annu Rev Genet 2008;

42 : 517-40.

3. El-Amraoui A, Petit C. Thérapie cellulaire dans l'oreille interne : nouveaux développements et perspectives. Med Sci (Paris) $2010 ; 26: 981-5$.

4. Amonlirdviman K, Khare NA, Tree DR, et al. Mathematical modeling of planar cell polarity to understand domineering nonautonomy. Science 2005 ; $307: 423-6$.

5. Tree DR, Ma D, Axelrod JD. A three-tiered mechanism for regulation of planar cell polarity. Semin Cell Dev Biol 2002 ; 13 : 217-24

6. Tree DR, Shulman JM, Rousset R, et al. Prickle mediates feedback amplification to generate asymmetric planar cell polarity signaling. Cell 2002 ; $109: 371-81$

7. Aigouy B, Farhadifar R, Staple DB, et al. Cell flow reorients the axis of planar polarity in the wing epithelium of Drosophila. Cell 2010 ; 142 : 773-86.

8. Classen AK, Anderson KI, Marois $\varepsilon$, Eaton S. Hexagonal packing of Drosophila wing epithelial cells by the planar cell polarity pathway. Dev Cell 2005 ; 9 : 805-17.

9. De Gennes PG, Prost J. The physics of liquid crystals, $2^{\mathrm{e}}$ ed. Gloucestershire, UK : Clarendon Press, 1993.

10. Joanny JF, Julicher F, Kruse K, Prost J. Hydrodynamic theory for multi-component active polar gels. NJ Phys $2007 ; 9: 422$.

\section{NOUVELLE}

\section{Sauver ou tuer}

L'ambivalence

des récepteurs des neurotrophines

Vincent Bischoff, Vassiliki Nikoletopoulou, Yves-Alain Barde
Biozentrum-University of Basel,

Klingelbergstrasse 50/70,

CH-4056 Bâle, Suisse.

vincent.bischoff@unibas.ch

après la découverte, il y a une cinquantaine d'années, d'une molécule désignée nerve growth factor (NGF) [2]. Des expériences aussi précoces que convaincantes ont en effet permis de montrer que le développement du système nerveux périphérique (SNP), en particulier la survie de la plupart des neurones sympathiques et sensoriels nouvellement différenciés, requièrent la présence de NGF. Au cours des décennies suivantes, il est cependant apparu que la survie d'une grande majorité de neurones du système nerveux central (SNC) était indépendante de la présence de facteurs trophiques de type NGF [3]. Trois autres molécules proches

\section{Rôle et mode d'action des neurotrophines \\ Chez les vertébrés, le contrôle du nombre de cellules pendant le développement du système nerveux obéit à des mécanismes que l'on pensait en grande partie résolus}

> Au cours du développement des vertébrés, l'homéostasie des différents tissus, et en particulier le contrôle du nombre de cellules, sont assurés par des voies de signalisation régulant la survie et la mort cellulaires. Les mêmes mécanismes sont souvent impliqués dans des processus pathologiques, comme dans les tissus matures. La connaissance des voies moléculaires permettant de contrôler important, tant pour la compréhension des mécanismes développementaux que pour celle des voies modulant la cancérogenèse. Certains de ces processus fondamentaux, notamment la mort cellulaire programmée,

sont maintenant bien compris grâce en particulier à l'apport de la génétique et nématode C. elegans [1]. En revanche, concernant le développement du systeme nerveux des vertébrés, l'absence laires adéquats fait qu'il reste beaucoup laires adéquats fait qu'il reste beaucoup à découvrir. 